4. Fried, D. L. t. opt. Soc. Am. 55, 1427-1435 (1965).

5. O'Neill, E. L. Introduction to Statistical Optics, 87-88 (Addison-Wesley, Reading, Mass., 1963)

6. Hardy, J. W. Proc. Soc. Photo-instrum. Engrs 1114, 2-13 (1989),

7. Roddier, F. Appl. Opt. 27, 1223-1225 (1988).

8. Smithson, R. C. Lockheed Horizon 28, 24-31 (1990),

9. Merkle, F. ESO Conf. Very Large Telescopes and Their Instrumentation Vol. 2 (ed. Ulrich, M-H.) 656 (European Southern Observatory, Garching bei Munchen, 1988).

10. Paxman, R. G. \& Fienup, J. R. J. opt. Soc. Am. A5, 914-923 (1988).

11. Hege, E. K., Beckers, J. M., Strittmatter, P. A. \& McCarthy, D. W. Appl. Opt. 24, 2565-2576 (1985).

12. McCarthy, D. W., Mcleod, B. A. \& Barlow, D. Proc. Soc. Photo-instrum. Engrs 1237, 496-507 (1990).

13. Born, M. \& Wolf, E. Principles of Optics 6th edn, 416 (Pergamon, Oxford, 1984).

14. Chaffee, F. H. \& Cromwell, R. H. Proc. Soc. Photo-instrum. Engrs 1236, $13-17$ (1990).

15. Cromwell, R. H., Haemmerle, V. R. \& Woolf, N. J. Proc. Soc. Photo-instrum. Engrs 1236, 520-529 (1990).

16. Lippmann, R. P. IEEE Acoustics, Speech, and Signal Processing Mag., 4-22 (April, 1987).

17. Rumelhart, D. E., Hinton, G. E. \& Williams, R. J., Parallel Distributed Processing Vol. 1 (eds Rumelhart, D. E. \& McClelland, J. L.) 318-362 (MIT Press, 1986)

18. Allen, C. W. Astrophysical Quantities 3rd edn, 244 (Athlone, London, 1973).

ACKNOWLEDGEMENTS. We thank R. Fugate for encouragement. This work was supported in part by the US Air Force Weapons Laboratory.

\section{An upper limit to violations of the Pauli exclusion principle}

\section{Kekez*, A. Ljubičic* \& B. A. Logan $\dagger$}

\author{
* Ruder Bošković Institute, PO Box 1016, 41001 Zagreb, Croatia, \\ Yugoslavia \\ † Ottawa-Carleton Institute of Physics, University of Ottawa, Ottawa, \\ Ontario, Canada, K1N 6N5
}

THE Pauli exclusion principle was formulated to explain the regularities of both the Periodic Table and atomic spectra ${ }^{1}$, and has a crucial role in our understanding of the structure and properties of atoms and nuclei. Despite its enormous success the absolute validity of this principle is still open to question and some recent theoretical analyses have suggested that small violations are possible. This has inspired several experimental tests of its validity. Here we consider the possibility of a $\boldsymbol{\beta}$-decay process that would violate the Pauli exclusion principle and use existing data from an underground laboratory to place a severe limit on the decay probability, and on possible violations of the Pauli exclusion principle.

In an extension to the field-theory analysis by Ignatiev and Kuzmin $^{2,3}$ of a single oscillator, Greenberg and Mohapatra ${ }^{4}$ developed a theory that allows for small violations of the Pauli exclusion principle (PEP). The analysis, to some extent a development of some ideas due to Green ${ }^{5}$, involves trilinear commutation relations that include a small parameter $\beta \ll 1$; $\beta^{2} / 2$ is a measure of the violation of the PEP and is defined as the relative probability of finding two fermions in the same state. Govorkov ${ }^{6}$ showed that their theory predicted states with negative probabilities, however, and it was concluded that it would not be possible to construct a field theory for small PEP violations ${ }^{7}$. The possibility of 'infinite statistics' was not treated by Govorkov; in such statistics no assumption is made restricting the number of particles in symmetric or antisymmetric states. These statistics have been studied ${ }^{8-10}$, and Mohapatra ${ }^{9,10}$ has suggested that it may be possible to construct a new kind of field theory in which small violations of the PEP could be allowed.

Experimental tests of the validity of the PEP are of considerable interest and the status of the current and proposed activity has been reviewed recently ${ }^{11-13}$. Novikov et al. ${ }^{14}$ investigated PEP violations by using accelerator mass spectrometry to search for anomalous atoms with three electrons in the $\mathrm{K}$ shell and obtained a limit of $\beta^{2} / 2<2 \times 10^{-21}$. Ramberg and Snow ${ }^{15}$ placed an upper limit on $\beta^{2} / 2$ of $1.6 \times 10^{-26}$ by searching for $\mathrm{K}$-shell $\mathrm{X}$-ray emission when a current is passed through a copper strip; the X-rays would be emitted if the 'new' electrons cascade down to an already occupied $\mathrm{K}$ shell. Recently we investigated the non-paulian electron-capture decay of ${ }^{71} \mathrm{Ge}$ and deduced a limit of $\beta^{2} / 2 \leqslant 3 \times 10^{-12}$ for neutrons ${ }^{16}$. We also considered the possibility of non-paulian decays in stable nuclei through $\beta^{-}\left(\beta^{+}\right)$ decays of higher-shell neutrons (protons) directly into the lower proton (neutron) shell. It is well known that if the hamiltonian of a system is permutationally invariant, transitions to a filled shell are forbidden for reasons not related to the PEP. But these restrictions are limited to systems in which the number of a given fermion does not change. In the case of $\beta^{-}\left(\beta^{+}\right)$decay both the number of protons and neutrons changes and the general restrictions for transitions to a filled shell do not apply. In particular, we studied the non-paulian decays of ${ }^{127} I$ into the non-paulian nuclei ${ }^{127} \mathrm{Xe}$ or ${ }^{127} \mathrm{Te}$. We searched for $\beta^{-}\left(\beta^{+}\right)$ particles with a large $\mathrm{NaI}(\mathrm{Tl})$ detector and obtained a limit of $\beta^{2} / 2 \leqslant 2 \times 10^{-26}$.

A much more severe limit on $\beta^{2} / 2$ can be deduced using the existing data from underground laboratories. The liquid scintillation detector ${ }^{17,18}$ (LSD) of the Mont Blanc collaboration is particularly suitable. It has been in operation since 1984 in a cavity in a tunnel linking France and Italy, about $1,700 \mathrm{~m}$ below the surface $(5,200 \mathrm{~m}$ water-equivalent). The LSD contains 90 tons of liquid scintillator in 72 stainless steel containers, each with a volume of $1.5 \mathrm{~m}^{3}$. The density of the liquid scintillator $\mathrm{C}_{n} \mathrm{H}_{2 n+2}(\bar{n}=10)$ is $0.8 \mathrm{~g} \mathrm{~cm}^{-3}$ and there are $5 \times 10^{28}$ carbon atoms in each container.

In ${ }^{12} \mathrm{C}$ the $2 p$ shell is $\sim 20 \mathrm{MeV}$ above the $1 s$ shell. The most probable non-paulian transition is expected to be the firstforbidden $2 p \rightarrow 1 s \beta^{-}\left(\beta^{+}\right)$transition to ${ }^{12} \mathrm{~N}\left({ }^{12} \mathrm{~B}\right)$. For a conventional decay the $\log f t$ values for such first forbidden transitions range from 6 to 9 ; a conservative value of 9 corresponds to a half-life of $\sim 300 \mathrm{~s}$. In each container the Pauli-allowed rate $N_{\mathrm{P}}$ for a $\beta$-decay to a $1 s$ shell with a vacancy is then $1.7 \times 10^{26} \mathrm{~s}^{-1}$.

During a 75-day run only two events with energies $\geqslant 12 \mathrm{MeV}$ were recorded in the inner 7.2 tons of the $\mathrm{LSD}^{19}$. In 1988 more shielding was added and this new configuration (LSD-2) has reduced background at lower energies ${ }^{20}$; the background for $E \geqslant 12 \mathrm{MeV}$, however, did not decrease. The counting rate of $3.1 \times 10^{-7} \mathrm{~s}^{-1}$ for $E \geqslant 12 \mathrm{MeV}$ can be used as a limit on $N_{\mathrm{PV}}$, the rate of the PEP-violating decays of ${ }^{12} \mathrm{C}$ to the occupied shell of ${ }^{12} \mathrm{~N}\left({ }^{12} \mathrm{~B}\right)$. The corresponding $N_{\mathrm{P}}$ rate for 7.2 tons is $0.97 \times$ $10^{27} \mathrm{~s}^{-1}$. We expect about half of the non-paulian decays to emit $\beta$-particles with energies above the LSD bias level of $12 \mathrm{MeV}$. In our case $\beta^{2} / 2=\lambda_{\mathrm{PV}} / \lambda_{\mathrm{P}} \leqslant 2 N_{\mathrm{PV}} / N_{\mathrm{P}}=6.5 \times 10^{-34}$ where $\lambda_{\mathrm{PV}}$ and $\lambda_{\mathrm{P}}$ are the decay constants.

This is a very severe limit on $\beta^{2} / 2$ and is many orders of magnitude more restrictive than the other available experimental limits. The decay of ${ }^{12} \mathrm{C}$ is probably not an ideal candidate for studying PEP violations and it may be possible to improve the limit by studying the non-paulian decay of other nuclei in underground laboratories.

\footnotetext{
Received 9 July: accepted 2 October 1990

1. Pauli, W. Z. Phys. 31, 765-783 (1925).

2. Ignatiev, A. Yu. \& Kuzmin, V. A. Yardenaya Fiz. 46, 786-790 (1987)

3. Ignatiev, A. Yu. \& Kuzmin, V. A. Soviet J. nucl. Phys.46, 444-446 (1987)

4. Greenberg, O. W. \& Mohaptra, R. N. Phys. Rev, Lett. 59, 2507-2510 (1987)

5. Green, H. S. Phys. Rev. 90, 270-273 (1953).

6. Govorkov, A. B. Phys. Lett. A137, 7-10 (1989).

7reenberg, O. W. \& Mohapatra, R. N. Phys. Rev. Lett. 62, 712-714 (1989),

Greenberg, O. W. Phys. Rev. Lett. 64, 705-708 (1990)

9. Mohapatra, R. N. Physics Preprint 90-120 (Univ. of Maryland, 1990)

10. Mohopatra, R. N. Bull. Am. phys. Soc. 35, 981 (1990).

11. Okun, L. B. Festi-Val-Festschrift for Val Telegdi (ed. Winter, K.) 201-211 (Elsevier, Amsterdam, 1988).

12. Okun, L. B. Comments nucl. Particle Phys. 19, 99-115 (1989).

13. Greenberg, O. W. \& Mohapatra, R. N. Phys. Rev. D39, 2032-2038 (1989).

14. Novikov, V. M. et al. Phys. Lett. B240, 227-231 (1990)

15. Ramberg, E. \& Snow, G. A. Phys. Lett. B238,438-441 (1990)

16. Kekez, D., Ljubičic, A., Kaucić, S. \& Logan, B. A. Z. Phys. (in the press).

17. Badino, G. et al. Nuovo Cim. C7, 573-590 (1984)

18. Aglietta, M. et al. Nuovo Cim C9, 185-195 (1986).

19. Aglietta $M$ et al. Proc Int Symp on Weak and Electromagnetic interactions in Nuclei (ed Klapdor

H. v) 741-744 (Spring

20. Aglietta, M. et al. Nucl. Phys. B14, 204-209 (1990)
}

ACKNOWLEDGEMENTS. We thank D. Dehler for discussions and O. Saavedra for a personal communication. B.A.L. thanks the Naturat Sciences and Engineering Research Council of Canada for support. 